\title{
LA CogNZIone del Dolore: ESPERIENZa di Traduzione e Forme di LetTura
}

\author{
Fabrizio Rusconi ${ }^{*}$ \\ ANDREA Lombardi * *
}

\begin{abstract}
Questo studio prende le mosse da una riflessione di Giacomo Leopardi sulla traduzione, annotata nel suo Zibaldone e nella quale si faceva menzione dell'impressione originaria che un buon traduttore deve provare a riprodurre nel testo tradotto. A partire da alcuni esempi concreti, si è tentato di comprendere come un'intera tradizione si sia allontanata o avvicinata a questa esigenza di traduzione e quale spazio essa abbia nell'attuale riflessione sul tema. Abbiamo infine analizzato la traduzione brasiliana della Cognizione del dolore di Carlo Emilio Gadda per mettere in luce come una corretta valutazione della specificità del testo, condotta sulla base delle impressioni di lettura suscitate nei lettori ad esso coevi, ma anche posteriori, avrebbe sicuramente giovato nella corretta valutazione delle scelte operate in traduzione. In conclusione dimostriamo, sulla base di una comparazione tra testo originale e testo tradotto, come questa consapevolezza, nel traduttore, non sempre esista o sia tenuta nel giusto conto, e come questa mancanza produca distorsioni e abbagli.
\end{abstract}

PAROLE CHIAVE: traduzione; impressione di lettura; Carlo Emilio Gadda; $L a$ Cognizione del Dolore; esperienza originaria.

RESUMO: Este estudo nasce de uma reflexão de Giacomo Leopardi sobre a tradução, anotada em seu Zibaldone, na qual se mencionava a impressão original que um bom tradutor deve tentar reproduzir no texto traduzido. A partir de alguns exemplos concretos, tentou-se compreender como uma tradição inteira se afastou ou se aproximou dessa exigência de tradução e que espaço ela teria na atual reflexão sobre o tema. Enfim, analisamos a tradução brasileira do Cognizione del dolore de

\footnotetext{
* Universidade Federal do Rio de Janeiro, Rio de Janeiro, RJ (Brasil) fabriziorusconi@gmail.com

** Universidade Federal do Rio de Janeiro, Rio de Janeiro, RJ (Brasil) lombardi.andrea@gmail.com
} 
Carlo Emilio Gadda para esclarecer como uma correta avaliação da especificação do texto, conduzida na base das impressões de leitura suscitadas nos leitores a ele coevos, mas também posteriores, certamente teria sido benéfica na correta avaliação das escolhas realizadas na tradução. Na conclusão demonstramos, com base em uma comparação entre texto original e texto traduzido, como essa consciência nem sempre existe no tradutor ou é considerada como deveria, e como essa falta produz distorções e enganos.

PALAVRAS-CHAVE: tradução; impressão de leitura; Carlo Emilio Gadda; Cognizione del dolore; experiência original.

ABSTRACT: This study is based on a reflection of Giacomo Leopardi on translation, annotated in his book Zibaldone, which mentioned that a good translator must try to reproduce the original idea in the translated text. From a few concrete examples, we tried to understand how a whole tradition turned away or approached this translation requirement and what space it would have in the current reflection on translation. Finally, we analyzed the Brazilian Portuguese translation of Cognizione del dolore by Carlo Emilio Gadda to clarify how a review of the specification of the text, conducted on the basis of reading impressions instilled in readers at the time or subsequent, certainly would have been beneficial in the correct assessment of the choices made in the translation. In the conclusion we showed, based on a comparison between the original and the translated text, that this awareness is not always considered as it should be by the translator, producing distortions and mistakes.

KEYWORDS: Translation; reading impression; Carlo Emilio Gadda; Cognizione del dolore; original experience. 
"Ya la traducción es en cierto sentido una concretización que lleva a cabo el traductor. La repercusión de la obra entre lectores y críticos en el medio extranjero difiere con gran frecuencia de la repercusión en el medio de origen, puesto que también la norma es diferente" (Vodicka, La historia literaria: sus problemas y tareas)

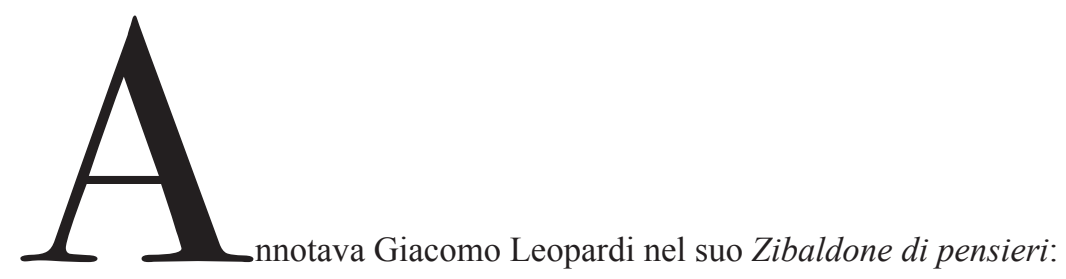

Un'osservazione importantissima intorno alle traduzioni, e che non so se altri abbia fatta, e di cui non ho in mente alcuno che abbia profittato, è questa. Molte volte noi troviamo nell'autore che traduciamo p.e. greco, un composto una parola 
che ci pare ardita, e nel renderla ci studiamo di trovargliene una che equivalga, e fatto questo siamo contenti. Ma spessissimo quel tal composto o parola comechè sia, non solamente era ardita, ma l'autore la formava allora a bella posta, e però nei lettori greci faceva quell'impressione e risaltava nello scritto come fanno le parole nuove di zecca, e come in noi italiani fanno quelle tante parole dell'Alfieri p.e. spiemontizzate ec. ec. Onde tu che traduci, posto ancora che abbi trovato una parola corrispondentissima proprissima equivalentissima, tuttavia non hai fatto niente se questa parola non è nuova e non fa in noi quell'impressione che faceva ne' greci. (LEOPARDI, 2007, p. 136).

Leopardi intuì molto prima di altri l'importanza che la ricezione estetica di un testo, dentro l'orizzonte d'attesa di lettori storicamente determinati, ha nella pratica letteraria, anche quella traduttiva, ossia l'effetto che determinate scelte di lingua, stile e sintassi provocano sul lettore coevo al testo da tradurre. Abbiamo usato termini presi a prestito dalle teorie novecentesche che più si sono occupate degli effetti del testo sui lettori. Eredi della tradizione critica e poetica novecentesca, con particolare enfasi, ai fini del nostro discorso, sull'ultima fase della scuola di Praga, i cui risultati saranno proficuamente ripresi e elaborati dalla Rezeptionsästhetik, sia dell'ermeneutica, possiamo facilmente capire la novità concettuale che Leopardi introdusse con questa annotazione. Attualizzandolo in termini concettuali tutti novecenteschi, il pensiero leopardiano può essere così espresso:

Nell'ambito della letteratura, il "circuito segmentato" crea le condizioni per una illimitata distanza temporale (storica), spaziale o culturale tra l'autore e i suoi (attuali o potenziali) riceventi. La variabilità della distanza genera rilevanti indeterminatezze, tensioni, discordanze semantiche. (DOLEZEL, 1990, p. 211).

Si pone così il problema di "catene di trasmissione" particolarmente complesse dalle quali i testi letterari necessariamente passano. È proprio l'esistenza di questa specificità nei meccanismi di trasmissione/ricezione del testo letterario, che il buon traduttore deve saper intuire nei suoi esiti pratici. Tradurre significherà non solo riprodurre l'adeguatezza lessicale di una parola ma altresì il suo effetto sul lettore coerentemente all'epoca in questione (o alle epoche): il testo deve rimandare necessariamente al contesto. Un buon traduttore, come un archeologo, dovrà mirare 
a ricostruire l'interezza di un mondo culturale - con le sue norme, i suoi valori -, giacché solo così sarà in grado di riprodurre l'esperienza di lettura originaria (Leopardi parla di impressione) che si è perduta dietro gli alfabeti. Ricostruire un clima culturale può avere una rilevanza estrema nel giustificare una scelta traduttiva anziché un'altra.

Umberto Eco nel suo saggio sulla traduzione intitolato Dire quasi la stessa cosa: esperienze di traduzione (2003) ritorna sulla questione sollevata da Leopardi e "riscoperta" da alcune scuole poetologiche novecentesche. Il problema di come determinate scelte lessicali fondino un'esperienza di lettura e come questa sia relativa a un'epoca, a una cultura, a una sensibilità, è esemplificato in tutta la sua complessità prendendo come esempio uno dei testi più universalmente tradotti: l'Ecclesiaste [o Qohelet]. "Vanitas vanitatum" è la locuzione al centro di un conflitto di interpretazioni che dura da secoli e che nel libro sapienziale apre e chiude il celebre discorso di Qohelet, probabilmente un calco linguistico dell'ebraico "havel havalim". Comparate nel saggio di Eco sono le traduzioni di Galbiati, Chouraqui, De Luca e due di Ceronetti. Così, se Galbiati si attiene alla madre di tutte le traduzioni che è ovviamente quella di San Girolamo e traduce alla lettera "vanità delle vanità! Tutto è vanità!", già Chouraqui inaugura uno scarto consistente rispetto a questa tradizione traducendo "fumée de fumée, tout est fumée" (ECO, 2003, p. 209). De Luca si può dire continui sulla scia di questa differenza, traducendo: "Spreco di sprechi il tutto è spreco". Infine le due traduzioni di Ceronetti, quello del 1970: "un infinito niente/ tutto è vuoto niente" e del 2001: "Fumo di fumi/ Tutto non è che fumo" (ECO, 2003, p. 210-211).

Comparativamente gli esempi ci mostrano come una parola o locuzione possa perdere nei secoli il suo significato originario e acquistarne altri, compatibilmente con l'avanzare dei costumi e delle sensibilità. Nel caso in esame, originariamente, il termine habèl/vanità funzionava poiché si riferiva "alle vane parvenze in senso metafisico, all'inconsistenza del tutto" (ECO, 2003, p. 212), mentre oggi quella traduzione potrebbe risultare fuorviante rimettendo semanticamente a una cura esagerata del proprio aspetto. Le traduzioni di Chouraqui, De Luca e Ceronetti sono altrettanti tentativi di uscire dall'impasse di una espressione banalizzante se intesa ricorrendo alla nostra sensibilità. Tuttavia, ci pare, queste traduzioni producono un eccesso di straniamento nel lettore contemporaneo che è immaginabile non rispetti l'impressione originaria, probabilmente più neutra, del testo ebraico e poi greco/latino. Discorso che vale anche per gli altri livelli testuali coinvolti dalla traduzione. Non solo il lessico, ma altresì la sintassi può richiedere una riflessione di questo tipo. Rimaniamo nell'ambito dell'esempio fatto, le varie traduzioni del Qohelet: 
Quanto all'ultimo verso, sia Chouraqui che De Luca scelgono una sintassi contorta (che non è né italiana né francese) proprio, e ancora una volta, per suggerire il profumo dello stile originario. [...] si vuole suscitare nel lettore la "nostalgia per l'originale". Che è poi, credo, quel sentimento del Das Fremde di cui parlava Humboldt. (ECO, 2003, p. 213).

Eppure, suggerire la "nostalgia per l'originale" può contraddire proprio l'esperienza di lettura originaria che di un simile sentimento non aveva naturalmente bisogno. Non siamo forse noi i cultori di un falso primitivismo, che si cela dietro la pretesa di un ritorno alla fonte, sempre più simili a quei collezionisti di maschere africane le quali usano per adornare i loro ricchi salotti europei?

Antoine Berman (2007), un grande teorico della traduzione, attentissimo ad evitare "le tendenze deformatrici" ${ }^{1}$ che operano sottotraccia in ogni traduttore, spesso inconsciamente, non spende una parola invero verso ciò che abbiamo chiamato l'esperienza originaria o l'impressione originaria al lettore di una data epoca confrontato con un dato testo. Essa, parimenti, esige di essere riprodotta, o almeno conosciuta, tanto quanto lo esigono le altre componenti formali immanenti al testo oggetto di traduzione. Tuttavia Berman si confronta con un problema diverso, ma speculare: riprodurre, fatalmente, l'impressione di una distanza che esisterebbe tra l'originale e il testo tradotto, distanza che dev'essere riassaporata nella buona traduzione affinché essa sia concepibile sempre e comunque come un'esperienza inusuale, esotica, straniante. " $O$ albergue do longínquo" è locuzione che rappresenta in forma lirica questo tipo di esperienza di allontanamento dal conosciuto, dall'usale che secondo l'autore dovrebbe guidare ogni traduzione che si rispetti. E tuttavia a noi pare che questo straniamento, questa vertigine di lontananza non sempre sia giustificata. La distanza storica che esiste tra noi e i classici è forse responsabile di un errore prospettico: pensare che la sensazione di straniamento, di insolito, di inconsueto sia originaria e non culturale e contingente. Equivoco che giustificherebbe un tipo di traduzione primitivista, per esempio il ceronettiano "fumo di fumi" (ECO, 2003, p. 211), o soltanto forzatamente eccentrica per l'uso che viene fatto di lessico, sintassi, tropi. In fondo è ragionevole pensare che proprio quei testi che a un lettore contemporaneo paiono esotici e difficili fossero parte di una consuetudine, corrispondendo a un'attesa - per quanto sicuramente colta - all'epoca della loro redazione. Questa considerazione può forse sembrare banale, ma le conseguenze evidentemente non lo sono: un testo può essere tradotto con più criterio tenendo conto di questo discrimine tra la nostra percezione e quella dei suoi antichi destinatari.

1 Antoine Berman elenca la presenza di una serie di tendenze deformatrici proprie di ogni traduttore, intrinsecamente culturali e quindi difficili da evitare, tali quali "a racionalização, a clarificação, o alongamento, o enobrecimento e a vulgarização, o empobrecimento qualitativo/quantitativo, a homogeneização, a destruição das redes significantes subjacentes, a destruição dos sistematismos textuais, a destruição das redes de linguagens vernaculares, a destruição das locuções e idiotismos, o apagamento das superposições de línguas" (BERMAN, 2007, p. 68). 
La nostra preoccupazione per una traduzione fedele non tanto e non solo al senso dell'originale quanto all'impressione che suscitò nei lettori ad esso contemporanei si rivela significativa per poter intendere e valutare gli effetti di un testo linguisticamente complesso e innovatore qual è la Cognizione del dolore di Carlo Emilio Gadda. Apparso originariamente e parzialmente nella rivista Letteratura, in sette numeri tra il 1938-1941, La cognizione, nel dopoguerra, divenne oggetto di una contrattazione tra l'autore, C. E. Gadda e lo stesso Giulio Einaudi. Della proposta si fece latore Vittorini nel 1952, chiamato l'anno prima da Giulio Einaudi a dirigere la collana "i Gettoni" di conio sperimentale. Varie vicissitudini ne ritardarono tuttavia l'uscita definitiva in volume, il che avvenne soltanto nel 1963 in altra sede: la collana einaudiana dei "Supercoralli". Quindi a una prima data - il finale degli anni 1930 - e a una collocazione in rivista, fa seguito una seconda uscita: questa sì in volume e per la già celebre e collaudata collana Einaudi. Evidentemente al traduttore consapevole e smaliziato si offrono due tempi di fruizione e plausibilmente due tipologie di pubblico, separate da due decenni abbondanti. Se per inciso, le prime impressioni di lettura - per lo più registrate in quell'anagrafe specifica che è la fortuna critica - nel continuum storico-critico confermano una somiglianza di reazioni, è auspicabile che il traduttore operi affinché anche nella nuova lingua e per un pubblico di contemporanei, questa impressione si conservi e sia riprodotta. Concretamente: se i primi lettori e i primi critici colsero la novità che la Cognizione e più in generale i testi gaddiani rappresentarono - Contini parlò di "espressionismo naturalistico" (CONTINI, [s.d.]) e di "pastiche" (CONTINI, 1989) ogni tentativo di traduzione che non si sforzasse di riprodurre l'esperienza che la lettura di un testo espressionista e linguisticamente sperimentale produce nei suoi lettori potrebbe risultare incoerente e difforme. È ciò che chiamerei lo scrupolo filologico del traduttore; scrupolo che può rivolgersi tanto a forme lessicali specifiche - come nella citazione leopardiana d'apertura quanto a figure o locuzioni la cui percezione sia storicamente mutata. Lo stesso scrupolo tuttavia si dovrà usare anche per quei testi che non abbiano ancora una storia critica e una loro specifica filologia: anche i testi della contemporaneità si iscrivono in un orizzonte di attesa e registrano forme di fruizione i cui effetti un buon traduttore considererà pertinenti al fine della propria traduzione, guardando agli effetti che questa avrà sui suoi lettori potenziali.

Passando dall'argomentazione teorica alla pratica dei testi, la Cognizione del dolore di Carlo Emilio Gadda, nella traduzione brasiliana, a cura di Mario Fondelli per i tipi della Rocco, intitolata $O$ Conhecimento da Dor (GADDA, 1998), non sembra confermare le attese di lettura che dell'opera sono venute fissandosi nel corso dei decenni. 
Difficoltà e incongruenze che mi sembrano presenti fin dal titolo. Tradurre la parola "Cognizione" con "Conoscenza" (in portoghese "Conhecimento") finisce per provocare una perdita semantica considerevole: il termine "cognizione" vibra di armoniche filosofiche e intertestuali che la scelta del termine più neutro sacrifica²:

Il termine cognizione è corrente nella trattatistica filosofica e psicologica sino almeno ai primi decenni del Novecento, non è fuori luogo individuare sul piano sintattico-lessicale moduli di una tradizione che potremmo definire storicomoralista. (MANZOTTI, 1996, p. 7).

Tradizione che va da Machiavelli, passando per Leopardi, e arrivando a Manzoni - tanto per dirne l'importanza. Inoltre, la stessa locuzione "Cognizione del dolore" appare numerose volte nel complesso dell'opera gaddiana 3 , tanto quanto nel romanzo in esame: "Due note venivano dai silenzi, quasi dallo spazio e dal tempo astratti, ritenute profonde come la cognizione del dolore" (GADDA, 2011, p. 732, corsivo mio).

E la ripetitività di un termine chiave, specie quanto esso sia inusuale, crea un vincolo percettivo con il lettore, oltre ad entrare di diritto nella simbologia e nella poetica dell'autore considerato. Lo stesso non può dirsi per la ripetizione di un termine comune e per ciò neutralizzato, consumato dall'uso.

Nella traduzione della Cognizione del dolore approntata per il mercato e per il lettore brasiliano, verifichiamo la presenza di una delle distorsioni più comuni del tradurre, i cui effetti Berman così riassumeva: "dinanzi a un'opera eterogenea - e il testo in prosa lo è quasi sempre - il traduttore ha la tendenza a unificare, a rendere omogeneo ciò che appartiene all'ordine del diverso" (BERMAN, 2007, p. 77, traduzione mia). Laddove naturalmente la prosa di Gadda, limitatamente alle parti in cui l'eterogeneità è ancora addomesticabile, corre questo pericolo e anzi lo vede realizzato. La tendenza ovviamente comporta non solo l'appiattimento di ciò che era eterogeneo in partenza, come anche l'alterazione dell'impressione che i lettori dell'originale ne hanno avuta e testimoniata.

Diamo ora qualche esempio concreto di questo pericolo, limitandoci ad analizzare uno specimen circoscritto di testo, però significativo per le difficoltà di traduzione ad esso peculiari.

2 Giuseppe Stellardi ha evidenziato che una simile difficoltà si è presentata nella traduzione inglese della Cognizione. Scrive Stellardi: "In effetti, a cominciare dal titolo, Acquainted with Grief, si conferma l'inevitabile sospetto di intraducibilità che il testo gaddiano spontaneamente suscita; acquainted, infatti può solo a stento pretendere a colmare il vuoto profondo e complesso lasciato da "cognizione" [...] Il titolo inglese non riproduce né l'effetto straniante, né l'oscuro germinare di senso imprecisabile che quello italiano immediatamente genera" (STELLARDI, 1995, p. 347-348).

3 Cfr. I Viaggi, la morte "la cognizione metafisica del male"; e un passo del capitolo IX: "si vede allora quanto sia vero che le grandi cognizioni non vengono all'intelletto degli uomini che per mezzo di grandi dolori" (MANZOTTI, 1996, p. 7). 
A pagina 665 della Cognizione si legge: "Le scranne erano tutte occupate, da ciò che normalmente le occupa" (GADDA, 2011). A pagina 113 dell'edizione tradotta è riportato: "As cadeiras já estavam todas ocupadas, por aquilo que normalmente as ocupa" (GADDA, 1998). A noi pare che quel "scranne" necessiti di un trattamento differenziato anche in traduzione: traducendo con il neutro "as cadeiras" si perde la specificità semantica, connotativa e direi anche densamente letteraria del termine originale. Ecco la definizione che del termine dà l'ottimo dizionario on-line della Treccani:

Scranna s. f. [dal longob. skranna «panca»]. - In origine, sedia dottorale, o seggio del giudice, di legno, con braccioli e con spalliera molto alta; con questo sign., la parola si è mantenuta (anche per l'influsso dantesco) nella locuz. fig. sedere a scranna, ergersi a giudice, assumere tono magistrale, soprattutto senza averne la competenza e l'autorità: Or tu chi se', che vuo' sedere a scranna, Per giudicar di lungi mille miglia Con la veduta corta d'una spanna? (Dante). (SCRANNA, [s.d.]).

Tra il termine scelto, con il suo specifico timbro connotativo alto-mimetico e la rappresentazione della gretta e somaresca burocrazia militare delle cartelle cliniche, si interpone l'ammiccamento ironico del narratore verso i suoi lettori. Quelle scranne sono davvero molto basse e precarie nella realtà dell'ospedale di Pastrufazio ${ }^{4}$, tantoché, come ironizza il narratore, in quei "locali di scrittura" si riversano "sempre nuovi apporti, di sempre più fattiva ortografia e ortopedia" (GADDA, 2011, p. 665).

Un altro esempio, forse più pesante, di equivoco terminologico si trova a pagina 114 del testo tradotto (GADDA, 1998). Qui l'espressione "o rosto todo esburacado pela acne" del giovane furiere, assistente del colonnello Di Pascuale, traduce "la faccia tutta imbitorzolata da un'acne" (GADDA, 2011, p. 666). Sennonché, "esburacado" ("bucherellato", in italiano) è concetto addirittura opposto rispetto alla faccia "imbitorzolata". Il bitorzolo infatti, sempre ricorrendo al prezioso ausilio del dizionario on-line Treccani, sarebbe una "piccola prominenza sulla superficie della pelle" (BITORZOLO, [s.d.]): quindi non un buco, né una serie di buchi che sono semmai ciò che resta nell'adulto di un'acne giovanile. Altra espressione con la quale il traduttore insiste nell'equivoco suddetto la si trova a pagina 114, dove sempre riferendosi al volto del furiere, si dice: "aquela sua primavera di crateras" (GADDA, 1998). "Crateras", al posto di "bitorzoli” - “con quella sua primavera di bitorzoli” (GADDA, 2011, p. 666) -,

4 L'estratto tratto dalle ultime pagine della prima parte della Cognizione è ambientato nell'ospedale militare di Pastrufazio. I protagonisti principali della scena sono: anonimamente, i soldati degenti e il personale di servizio che ha il compito di osservarli e redigere le cartelle cliniche, quindi il colonnello medico di origini napoletane Di Pascuale e un certo Gaetano Palumbo, invalido di guerra che allega una sordità permanente e totale a entrambi i timpani in seguito all'esplosione di una granata durante la guerra: nel testo la guerra tra il Maradagal e il Parapagal. Il Di Pascuale è incaricato di stabilire se l'invalidità è reale o meno e qualora fosse comprovata, dopo debito periodo di controllo e valutazione delle reazioni del degente, concedere licenza e pensione di invalidità. 
modifica in senso iperbolico l'originale, persistendo nell'imprecisione dermatologica. "Bitorzoli" sarebbe al più traducibile con "verrucas". E infatti, violando ogni coerenza testuale, alla terza occorrenza della parola "bitorzoli", troviamo finalmente il giusto corrispettivo in portoghese: "O rosto do furriel, ligeiramente menos amarelo do que de costume sob a escumadeira avinhada das verrucas" (GADDA, 1998, p. 115), che nel testo originale riporta: "La faccia del furiere, un po' meno gialla del solito sotto alla schiumarola avvinata dei bitorzoli” (GADDA, 2011, p. 667).

Nella stessa pagina, segno della difficoltà e densità del testo nel suo complesso, troviamo un'altra semplificazione frasale che abbassa il grado di espressività rispetto all'originale: "Il colonnello Di Pascuale si voltò come l'avesse mozzicato un aspide" (GADDA, 2011, p. 667) diventa nel testo tradotto: "O coronel Di Pascuale virou-se como que mordido por uma víbora". Iniziamo dalla sostituzione più vistosa: il termine "víbora", in italiano "vipera", nomina un comunissimo serpente velenoso, ben diverso insomma dall'aspide che è forma più nobile, avallata da tutta una tradizione tragica, forte della sua iconografia storico-artistica: è l'aspide di Cleopatra, per esempio, o quella biblica. Scelta per altro facile, dato che il portoghese conosce lo stesso vocabolo per designare lo stesso referente: áspide appunto. Per quanto riguarda il verbo "mozzicare", tradotto con "mordido", la questione si fa più complessa. Mozzicare è infatti voce centro-meridionale, che ha l'intuito di avvicinarsi stilisticamente al personaggio citato: il napoletano Di Pascuale. Usando il verbo morsicare a cui manca ogni connotazione regionale, il traduttore sembra ignorare la sottile ironia di chi sta facendo il verso al personaggio. Tuttavia, la problematica del tradurre il dialetto è molto delicata e complessa. La traduzione di Fondelli sceglie di uniformare le battute in dialetto napoletano del Di Pascuale, che impiantate in un contesto come quello brasiliano sfiora l'intraducibilità. Per quanto legittima e sensata sia questa scelta, condanna il testo tradotto a perdere qualcosa di essenziale. La dialettalità rimane un tratto importante nella caratterizzazione del personaggio Di Pascuale. Questi è insomma il tipico esemplare del partenopeo: solerte, furbo, disincantato e rude ma, ciononostante, provvisto di una malizia quasi femminile.

Continuando sul testo, incontriamo un altro passaggio in cui la liricità dell'originale, costruita su una sequenza allitterativa, non è preservata - e forse non avrebbe potuto esserlo: "Collo: che appariva quasi bendato dalla bianca benda militare" (GADDA, 2011, p. 668).

Si faccia caso all'allitterazione delle labiali e delle dentali che tramite la sostanza lirica dell'enunciato innalza il referente, si parla dei morti in battaglia: be-ndato/bi-anche/be-nde. Ben-da-to da-lla ben-da. In traduzione è tutto più letterale e quindi più povero: "que parecia quase fechado pela branca gola militar" (GADDA, 1998, p. 116). 
La regolarizzazione di ciò che appartiene all'ordine del diverso, del non conforme, investe anche la punteggiatura, che in Gadda ha una funzione più ritmica e emotiva che sintattica. La violazione più consistente della norma ortografica nella Cognizione, come in altri testi dell'autore, si rileva nell'uso consecutivo dei due punti. Il traduttore, probabilmente per adempiere a un dovere di correttezza redazionale, non se l'è sentita di confermare una violazione tanto eclatante alle norme dell'interpunzione. Così, ogni qualvolta Gadda mette in fila la sua consueta serie di due punti, il traduttore normalizza sostituendolo (o sostituendoli) con punto e virgola. Vediamo qualche esempio: "Il colonnello Di Pascuale si voltò come l'avesse mozzicato un aspide: lo guardò: gli si avvicinò" (GADDA, 2011, p. 667), mentre in traduzione abbiamo: "O coronel Di Pascuale virou-se como que mordido por uma víbora: observou-o; aproximou-se dele" (GADDA, 1998, p. 115).

O ancora:

Il colonnello li prese, si accostò al tavolo, intinse la penna, si curvò, e firmò distrattamente: con l'animo sempre alla disputa, era evidente, e al collega: che gli seguitava a parlare tuttavia, angustiandolo di continue obbiezioni (a quanto già affermato dal Di Pascuale): un cane che non demorde. (GADDA, 2011, p. 667).

O coronel pegou-os, chegou-se à mesa, molhou a pena, curvou-se e assinou displicentemente: pensando na disputa, era evidente, e no colega que ainda seguia falando, angustiando-o com contínuas objeções (àquilo já afirmado pelo Di Pascuale) um cão que não larga a presa. (GADDA, 1998, p. 115).

Per concludere, se è vero che il testo di Gadda confermando la sua natura espressionista è un intrico di difficoltà praticamente insormontabili, è altresì vero che la traduzione analizzata, per quanto semplificata e parziale, riesce comunque a veicolare una certa conoscenza dell'originale. Insomma, Gadda nonostante la ricchezza e complessità della sua scrittura, o forse proprio grazie ad essa, sopravvive anche alle sue "traduzioni". Cartina al tornasole di quanto una traduzione possa considerarsi riuscita sarebbe provare a confrontare le esperienze di lettura e le rispettive impressioni tra lettori appartenenti a culture, climi, letterature e lingue diverse. Senza pretendere la precisione delle scienze esatte, perché come ci ricorda Umberto Eco "le opere letterarie ci pongono di fronte alle ambiguità del linguaggio e della vita" (ECO, 2002, p. 55), questo 
confronto tra impressioni e esperienze potrebbe riuscire profittevole al traduttore coscienzioso che abbia chiara la dimensione etica del proprio operare.

\section{Riferimenti bibliografici}

BERMAN, A. A tradução e a letra ou o albergue do longínquo. Rio de Janeiro: 7 Letras, 2007.

BITORZOLO. In Treccani, la cultura italiana. [s.d.]. http://www.treccani.it/vocabolario/ bitorzolo_\%28Sinonimi-e-Contrari\%29/ Acesso: 12/12/2016.

CONTINI, G. Introduzione alla Cognizione del dolore. In The Edimburgh Jornal of Gadda Studies, Edimburgh, [s.d.]. http://bit.ly/2hdB08N Acesso: 03/03/2015.

. Quarant'anni d'amicizia. Scritti su Carlo Emilio Gadda (1934-1988). Torino: Einaudi, 1989.

DOLEZEL, L. Poetica occidentale. A cura di Adelheid Conte. Torino: Einaudi, 1990.

ECO, U. Dire quasi la stessa cosa. Esperienze di traduzione. Milano: Bompiani, 2003.

. Sulla letteratura. Milano: Bompiani, 2002.

GADDA, C. E. O Conhecimento da Dor. Rio de Janeiro: Rocco, 1998.

. Opere. Romanzi e racconti. Vol. 1. A cura di Raffaella Rodondi, Guido Lucchini, Emilio Manzotti. Milano: Garzanti, 2011.

LEOPARDI, G. Zibaldone di pensieri. Milano: Mondadori, 2007.

MANZOTTI, E. Letteratura italiana. Le opere. Vol. IV.II. A cura di Alberto Asor Rosa. Torino: Einaudi, 1996.

SCRANNA. In Treccani, la cultura italiana. [s.d.]. http://www.treccani.it/vocabolario/scranna/ Acesso: 12/12/2016.

STELlardi, G. Gadda tradotto. In TERZOLI, M. A. (ed.). Le lingue di Gadda. Atti del Convegno di Basilea, 10-12 dicembre 1993. Roma: Salerno, 1995, p. 343-362.

Recebido em 03/04/2016

Aprovado em 08/06/2016 